\title{
METHODS FOR SOLVING THE STOCHASTIC POINT REACTOR KINETIC EQUATIONS*
}

\author{
EhSanur R. Quabili $\dagger$ and Muzaffer Karasulu $\ddagger$ \\ Department of Nuclear Engineering, The University of Michigan, Ann Arbor, Michigan 48109, U.S.A.
}

(Received 2 October 1978)

\begin{abstract}
In this paper we present two new methods to analyze the statistical properties of nonlinear outputs of a point reactor to stochastic non-white reactivity inputs. They are Bourret's approximation and logarithmic linearization. The results have been compared with the exact results, previously obtained in the case of Gaussian white reactivity input. It was found that when the reactivity noise has short correlation time, Bourret's approximation should be recommended because it yields results superior to those yielded by logarithmic linearization. When the correlation time is long, Bourret's approximation is not valid, but in that case, if one can assume the reactivity noise to be Gaussian, one may use the logarithmic linearization.
\end{abstract}

\section{INTRODUCTION}

The importance of understanding fluctuations in power reactors is generally agreed upon nowadays. Saito (1974) and Williams (1974) emphasized this importance and their works contain a sufficiently complete list of related references. Noise in reactors can be described by conventional Point Reactor Kinetic Equations (PRKE) with fluctuation introduced in some of the parameters. Such equations may be referred to as Stochastic PRKE. Power reactor noise analysis may be viewed as study of a reactor's response to a stochastic reactivity or source input. The difficulty of solving Stochastic PRKE arises from the fact that they are nonlinear, i.e. the noise is 'parametric' or 'multiplicative' (in contrast to 'additive').

If the input noise is Gaussian white, the FokkerPlanck theory is useful to find the moments (Ariaratnam and Graefe, 1964, 1965) and has been applied by Williams (1969, 1971), Karmeshu and Bansal (1975), Akcasu and Karasulu (1976), Dutré and Debosscher (1977), and Karmeshu (1978). Although the fundamental (microscopic) processes like neutronic noise, vibration of control and fuel rods, thermal-hydraulic noise etc., which are the cause of the reactivity noise, themselves may possibly be white, the macroscopic reactivity noise itself is not white because of various time constants involved in the

\footnotetext{
* Based on some parts of the authors' doctoral theses.

† Permanent Address: Bangladesh Atomic Energy Commission, Institute of Nuclear Technology, Dacca, Bangladesh.

$\ddagger$ Permanent Address: Technical University of Istanbul, Institute for Nuclear Energy, Istanbul, Turkey.
}

reactor system. Also, the fundamental noises may be conjectured to be Gaussian but they are connected to reactivity in a nonlinear fashion and so the reactivity noise is non-Gaussian. This necessitates the extension of power reactor noise analysis to nonwhite reactivity noise input. Gotoh (1975), and Karmeshu and Bansal (1975) discussed non-white reactivity noise to some extent.

The solution of stochastic differential equations with non-Gaussian non-white parametric noise, in general, is not possible without some approximations; and such approximations are a current topic of statistical mechanics. Van Kampen (1976) reviewed the methods of solving stochastic differential equations and here we use one of those reviewed, viz. Bourret's approximation.

In Section 2, we present another alternative method based on logarithmic linearization. In Section 3, we describe the Bourret's approximation technique with some extension of Van Kampen's review, and then apply it to the Stochastic PRKE. A comparative study of the results obtained by these two methods and other previous works will be made in Section 4.

\section{STOCHASTIC POINT REACTOR KINETIC EQUATIONS}

The Stochastic PRKE, with one group of delayed neutron, can be written as

$$
\begin{aligned}
& \frac{\mathrm{d} P(t)}{\mathrm{d} t}=\left[K_{0}+k(t)-1\right] P(t)+\lambda C(t)+S_{0} \\
& \frac{\mathrm{d} C(t)}{\mathrm{d} t}=P(t)-\lambda C(t),
\end{aligned}
$$


where $P(t)$ and $C(t)$ are the reactor power and delayed neutron precursor density, respectively; $S_{0}$ is the external source; $\lambda$ is the decay constant of delayed neutron; and $K_{0}$ and $k(t)$ are, respectively, the sure and fluctuating part of the reactivity in dollars. The unit of time chosen here is the ratio of neutron lifetime to delayed neutron fraction, i.e. $\ell / \beta$. This equation is the same as the conventional PRKE with constant reactivity, $K_{0}$, except for the added fluctuating part $k(t)$ whose following statistics we suppose to be known.

$$
\langle k(t)\rangle=0 ; \quad\langle k(t) k(t+\tau)\rangle=\phi_{k}(\tau) .
$$

Besides the stationarity of $k(t)$ assumed above, other properties or restrictions, or special forms of $\phi_{k}(\tau)$ will be assumed later.

Now it is our purpose to determine the statistical properties such as moments, autocorrelation function and power spectral density of reactor power in terms of the statistical properties of $k(t)$. Although our analysis can be carried out with more than one delayed neutron group, we do not do so for the sake of simplicity. Only one group of delayed neutron keeps the results simple, enabling us to make transparent comparisons.

An integro-differential form of PRKE, which will be used in the logarithmic linearization method is given below.

$$
\begin{aligned}
\frac{\mathrm{d} P(t)}{\mathrm{d} t}= & {\left[K_{0}+k(t)-1\right] P(t) } \\
& +\int_{0}^{\infty} \mathrm{d} u D(u) P(t-u)+S_{0},
\end{aligned}
$$

where

$$
D(u)=\lambda \mathrm{e}^{-\lambda u} ; \quad \int_{0}^{\infty} \mathrm{d} u D(u)=1
$$

\section{ANALYSIS BY LOGARITHMIC LINEARIZATION}

We introduce

$$
P_{0} K_{0}+S_{0}=0
$$

and

$$
y(t)=\ln \left[P(t) / P_{0}\right] .
$$

Now eliminating $S_{0}$ by $P_{0} K_{0}$ and using the following approximations

$$
|y(t-u)-y(t)| \ll 1 \text { and }|y(t)| \ll 1,
$$

one may reduce (3) to

$$
\begin{aligned}
& \frac{\mathrm{d} y(t)}{\mathrm{d} t}+\left(1-K_{0}\right) y(t)-\int_{0}^{t} \mathrm{~d} u D(u) y(t-u) \\
&=k(t) ; t>0 .
\end{aligned}
$$

Since $|y(t-u)-y(t)|=|\ln P(t-u) / P(t)|$, the first approximation implies that the logarithm of relative power change over time intervals in which $D(u)$ is appreciable, is small; hence the name logarithmic linearization. This logarithmic linearization was successfully used before to find the response of a point reactor to deterministic inputs in the absence of an external source (Akcasu et al., 1971). To linearize the term associated with the source term, $|y(t)|$ is assumed to be small compared to unity and this is a relatively weak assumption when the source is small.

One can take the Laplace transform of (8), solve for $\tilde{Y}(s)$ and take the inverse Laplace transform (using the convolution theorem) to get

$$
y(t)=\int_{0}^{t} \mathrm{~d} u q(u) k(t-u),
$$

where $q(u)$ is the unit impulse response of the reactor which is obtained as the inverse Laplace transform of $Q(s)$ given by

$$
\begin{gathered}
Q(s)=(s+\lambda) /\left(s-\gamma_{1}\right)\left(s-\gamma_{2}\right) ; \\
q(t)=C_{1} \mathrm{e}^{\gamma_{1} t}+C_{2} \mathrm{e}^{\gamma_{2} t},
\end{gathered}
$$

where $\gamma_{1}$ and $\gamma_{2}$ are the solutions of the inhour equation,

$$
s^{2}+\left(1+\lambda-K_{0}\right) s-\lambda K_{0}=0 .
$$

Near criticality,

$$
\begin{gathered}
C_{2}=(1+\lambda)^{-1} ; \quad C_{1}=\lambda C_{2} ; \\
\gamma_{1}=-\left|K_{0}\right| ; \quad \gamma_{2}=-(1+\lambda) .
\end{gathered}
$$

Now we make an assumption- $-k(t)$ is a Gaussian process. This allows us the use of the following relation

$$
\left\langle\mathrm{e}^{\xi(t)}\right\rangle=\exp \left\{\langle\xi(t)\rangle+\frac{1}{2} \operatorname{Var}[\xi(t)]\right\} .
$$

Inserting (6) in (9) and using (13) we obtain the moments of $P(t)$ as follows:

$$
\begin{aligned}
\left\langle P^{m}(t)\right\rangle= & P_{0}^{m} \exp \left\{\langle m y(t)\rangle+\frac{1}{2} \operatorname{Var}[m y(t)]\right\} \\
= & P_{0}^{m} \exp \left\{\frac{m^{2}}{2} \int_{0}^{t} \mathrm{~d} u \int_{0}^{t} \mathrm{~d} \vartheta q(u) q(\vartheta)\right. \\
& \times\langle k(t-u) k(t-\vartheta)\rangle\} \\
= & P_{0}^{m} \exp \left\{\frac{m^{2}}{4 \pi} \int_{-\infty}^{+\infty} \mathrm{d} \omega G_{k}(\omega)\right. \\
& \left.\times\left|\int_{0}^{t} \mathrm{~d} u q(u) \mathrm{e}^{-i \omega \mu}\right|^{2}\right\},
\end{aligned}
$$

where $G_{k}(\omega)$ is the Fourier transform of $\phi_{k}(\tau)$, i.e. the power spectral density of reactivity. In the stationary state (as $t \rightarrow \infty$ ) we find

$$
\left\langle P^{m}\right\rangle=P_{0}^{m} \exp \left\{\frac{m^{2}}{4 \pi} \int_{-\infty}^{+\infty} \mathrm{d} \omega G_{k}(\omega)|Q(i \omega)|^{2}\right\} .
$$


Similarly we obtain the autocorrelation function of $P(t)$ in the stationary state by using

$$
\begin{aligned}
\lim _{t \rightarrow \infty}\langle P(t) P(t+\tau)\rangle= & \lim _{t \rightarrow \infty} P_{0}^{2} \exp \{\langle y(t)+y(t+\tau)\rangle \\
& \left.+\frac{1}{2} \operatorname{Var}[y(t)+y(t+\tau)]\right\}
\end{aligned}
$$

as

$$
\phi_{p}(\tau)=P_{0}^{2} \exp \left\{\frac{1}{2 \pi} \int_{-\infty}^{+\infty} \mathrm{d} \omega G_{k}(\omega)|Q(i \omega)|^{2}\left(1+\mathrm{e}^{i \omega \tau}\right)\right\} .
$$

The normalized autocovariance function can be found as

$$
\frac{C_{p}(\tau)}{C_{p}(0)}=\frac{\exp \left[C_{y}(\tau)\right]-1}{\exp \left[C_{y}(0)\right]-1}
$$

where $C_{y}(\tau)$ is defined as the autocovariance of $y(t)$ given by

$$
C_{y}(t, t+\tau)=\int_{0}^{\tau} \mathrm{d} u \int_{0}^{t+\tau} \mathrm{d} \vartheta q(u) q(\vartheta) \phi_{k}(u-\vartheta+\tau) .
$$

We evaluate (17) for white reactivity noise, i.e. $\phi_{k}(\tau)=G_{k} \delta(\tau)$ or $G_{k}(\omega)=G_{k}$. We substitute $(10)$ in (17), approximate $\exp \left(-\left|K_{0}\right| t\right)$ by $1-\left|K_{0}\right| t$ and then let $K_{0} \rightarrow 0$.

$$
\begin{aligned}
\frac{C_{p}(\tau)}{C_{p}(0)}= & \exp \left\{\frac { G _ { k } } { 2 } \left[-\left(\frac{\lambda}{1+\hat{\lambda}}\right)^{2} \tau-\frac{(1+2 \lambda)}{(1+\lambda)^{3}}\right.\right. \\
& \left.\left.\times\left(1-\mathrm{e}^{-(1+\lambda) \tau}\right)\right]\right\} .
\end{aligned}
$$

This was first obtained by Dutré (1964), starting from an initially critical reactor (without source) and with Gaussian white reactivity insertion. Thus, the results (15) and (16) are the generalized forms of the previous results, because there is no restriction that the reactivity noise be white. For example, suppose the reactivity inserted is a narrow-band noise centered about a frequency $\omega_{0}$, whose power spectral density is given by

$$
G_{k}(\omega)=\pi \sigma_{k}^{2}\left[\delta\left(\omega+\omega_{0}\right)+\delta\left(\omega-\omega_{0}\right)\right],
$$

where $\sigma_{k}^{2}$ is the variance. Substituting (20) in (15) and (16) one gets

$$
\left\langle P^{m}\right\rangle=P_{0}^{m} \exp \left\{\frac{m^{2}}{2} \sigma_{k}^{2}\left|Q\left(i \omega_{0}\right)\right|^{2}\right\}
$$

and

$$
\phi_{P}(\tau)=P_{0}^{2} \exp \left\{\sigma_{k}^{2}\left|Q\left(i \omega_{0}\right)\right|^{2}\left(1+\mathrm{e}^{i \omega_{0} \tau}\right)\right\},
$$

where $\left|Q\left(i \omega_{0}\right)\right|^{2}$ can be found explicitly from (10).

In the case where there is no external neutron source, it is impossible to obtain the stationary moments and autocorrelation of $P(t)$ directly from
(15) and (16). If there is no external source the starting equation (9) has to be replaced by

$$
y(t)=\int_{0}^{t} \mathrm{~d} u z(u) K(t-u),
$$

where $z(u)$ is equal to $q(u)$ given by (10) and (11), with $K_{0}=0$. The moments of $P(t)$ in the stationary state reduce to

$$
\begin{aligned}
\left\langle P^{m}(t)\right\rangle= & \lim _{t \rightarrow x} P_{0}^{m} \exp \left\{m K_{0} \int_{0}^{t} \mathrm{~d} u z(u)\right. \\
& +\frac{m^{2}}{4 \pi} \int_{-\infty}^{+x} \mathrm{~d} \omega G_{k}(\omega) \\
& \left.\times\left|\int_{0}^{t} \mathrm{~d} u z(u) \mathrm{e}^{-i \omega u}\right|^{2}\right\} \\
= & P_{0}^{m} \exp \left\{m\left(K_{0}+\frac{m}{2} G_{k}(0) z_{x}\right) z_{x} t+m K_{0} A_{\alpha}\right\}
\end{aligned}
$$

where

$$
\begin{gathered}
z_{x}=\lambda /(1+\lambda) ; \quad A_{x}=(1 / 1+\lambda)^{2} ; \\
\lim _{t \rightarrow x} \int_{0}^{t} \mathrm{~d} u z(u)=z_{x} t+A_{x} .
\end{gathered}
$$

Here we have used the following asymptotic relation:

$$
\lim _{t \rightarrow \infty} \int_{-x}^{+\infty} \mathrm{d} \omega G_{k}(\omega)\left|\int_{0}^{t} \mathrm{~d} u z(u) \mathrm{e}^{-i \omega u}\right|^{2}=\frac{1}{2 \pi} G_{k}(0) z_{x x}^{2} t .
$$

It is seen that $\left\langle P^{m}(t)\right\rangle$ remains bounded as $t \rightarrow \infty$, if the mean of the reactivity $K_{0}$ is related to its power spectral density at $\omega=0$ by

$$
K_{0}+\frac{m}{2} G_{k}(0) z_{x}=0 .
$$

Otherwise, all the moments either increase or decay exponentially for large values of time. If $G_{k}(0)=0$ all the moments attain finite values when $K_{0}=0$, otherwise we always need to have $K_{0} \neq 0$ for stationarity, contrary to the case with an external source. When $G_{k}(0)=K_{0}=0,(25)$ is satisfied for all values of $m$ and all the moments attain finite values as $t \rightarrow \infty$. For example, suppose the power spectral density of reactivity is again given by (20). One can insert it in (24) and obtain the stationary moments as

$$
\left\langle P^{m}(t)\right\rangle=P_{r}^{m} \exp \left\{m^{2} \frac{\sigma_{k}^{2} z_{\infty}}{\omega_{0}}\left|Z\left(i \omega_{0}\right)\right| \sin \left(\omega_{0} t+\psi\right)\right\},
$$

where $P_{r}^{m}$ is defined as

$$
P_{r}^{m}=P_{0}^{m} \exp \left\{\frac{m^{2}}{2} \sigma_{k}^{2}\left[\left|Z\left(i \omega_{0}\right)\right|^{2}+\left(z_{\infty} / \omega_{0}\right)^{2}\right]\right\}
$$

and $\psi$ is the argument of $Z\left(i \omega_{0}\right)$.

When $K_{0}=0$ and if (25) is satisfied with $m=1$, 
the mean of $P(t)$ approaches a stationary value as $t \rightarrow \infty$, but its mean square diverges, i.e.

$\lim _{t \rightarrow \infty}\left\{\begin{array}{l}\left\langle P(t)=P_{0} \mathrm{e}^{K_{0} A_{x}}\right. \\ \left\langle P^{2}(t)\right\rangle \rightarrow \infty\end{array}\right\} ; \quad K_{0}+\frac{1}{2} G_{k}(0) z_{\infty}=0$.

On the other hand, if (25) is satisfied with $m=2$, the mean square attains a stationary value as $t \rightarrow \infty$ but the mean decays exponentially, i.e.

$\lim _{t \rightarrow \infty}\left\{\begin{array}{l}\left\langle P^{2}(t)\right\rangle=P_{0}^{2} \mathrm{e}^{2 K_{0}} \mathbf{z}^{A_{\infty}} \\ \langle P(t)\rangle \rightarrow 0\end{array}\right\} ; \quad K_{0}+G_{k}(0) z_{\infty}=0$.

This somewhat pathological situation is a consequence of the fact that the reactor is considered to be critical prior to $t=0$. One may point out that this difficulty does not arise if there were an external neutron source present.

The autocorrelation function of the power to a Gaussian non-white reactivity can be obtained as follows:

$$
\begin{aligned}
\phi_{p}(t, t+\tau)= & P_{0}^{2} \exp \left\{K_{0}\left[2 z_{x} t+z_{\infty} \tau+A(t)+A(t+\tau)\right]\right. \\
& +\frac{1}{4 \pi}\left[\int_{-\infty}^{+\infty} \mathrm{d} \omega G_{k}(\omega)\right. \\
& \times\left\{\left|\int_{0}^{t} \mathrm{~d} u z(u) \mathrm{e}^{-i \omega \omega u}\right|^{2}+\left|\int_{0}^{t+\tau} \mathrm{d} u z(u) \mathrm{e}^{-i_{\omega} \omega}\right|^{2}\right. \\
& \left.\left.\left.+\int_{0}^{t} \mathrm{~d} u \int_{0}^{t+\tau} \mathrm{d} \vartheta z(u) z(\vartheta) \mathrm{e}^{-i \omega(u-\vartheta+\tau)}\right\}\right]\right\} .
\end{aligned}
$$

This result is valid for any finite $t$ and for any Gaussian reactivity insertion. If we consider its behavior as $t \rightarrow \infty$ for a fixed $\tau$, we again face the question of convergence of the mean-square value of $P(t)$. Since $|\langle P(t) P(t+\tau)\rangle| \leq\left|\left\langle P^{2}(t)\right\rangle\left\langle P^{2}(t+\tau)\right\rangle\right|^{\frac{1}{2}}$, the autocorrelation remains finite as $t \rightarrow \infty$ for a fixed $\tau$ if $\left\langle P^{2}(t)\right\rangle$ does so, i.e. if $K_{0}+G_{k}(0) z_{\infty}=0$ [cf. (27)]. Since $\langle P(t)\rangle \rightarrow 0$, when this condition is satisfied, the autocorrelation and autocovariance of $P(t)$ are identical in the limit of $t \rightarrow \infty$, i.e.

$$
\begin{gathered}
\left\{\lim _{t \rightarrow \infty} \phi_{p}(t, t+\tau)=\lim _{t \rightarrow \infty} C_{p}(t, t+\tau)\right\} ; \\
K_{0}+G_{k}(0) z_{\infty}=0 .
\end{gathered}
$$

Therefore, the stationary normalized autocovariance function of $P(t)$ can be obtained from (28) and (24) as

$$
\begin{aligned}
\frac{C_{p}(\tau)}{C_{p}(0)} & =\lim _{t \rightarrow \infty} \frac{\langle P(t) P(t+\tau)\rangle}{\left[\left\langle P^{2}(t)\right\rangle\left\langle P^{2}(t+\tau)\right\rangle\right]^{1 / 2}} \\
& =\lim _{t \rightarrow \infty} \exp \left\{\int_{0}^{t} \mathrm{~d} u \int_{0}^{t+\tau} \mathrm{d} \vartheta z(u) z(\vartheta) \phi_{k}(u-\vartheta+\tau)\right.
\end{aligned}
$$

$$
\begin{aligned}
& -\frac{1}{2}\left[\left(\int_{0}^{t} \mathrm{~d} u \int_{0}^{t} \mathrm{~d} \vartheta+\int_{0}^{t+\tau} \mathrm{d} u \int_{0}^{t+\tau} \mathrm{d} \vartheta\right)\right. \\
& \left.\left.\times\left(z(u) z(\vartheta) \phi_{k}(u-\vartheta)\right)\right]\right\} .
\end{aligned}
$$

This result is again a generalized form of that obtained previously by Dutré (1964) which is given in (19). Indeed, if one substitutes $\phi_{k}(\tau)=G_{k} \delta(\tau)$ and $z(t)=z_{\infty}+\left[\left(z_{\infty} / \lambda\right] / \exp [-(1+\lambda) t]\right.$ in (29), one gets (19) which was obtained before, by making the assumptions $\exp \left(-\left|K_{0}\right| t\right) \simeq 1-\left|K_{0}\right| t$ and then $K_{0} \rightarrow 0$ in the case with a source.

\section{ANALYSIS BY BOURRET'S APPROXIMATION}

Bourret's approximation was developed by Redfield (1965), Howe (1971) and Bourret (1962), and reviewed by Van Kampen (1976). Here we follow Van Kampen's review essentially and make some extension to accommodate inhomogenous differential equations, in general. We start with the following differential equation

$$
\frac{\mathrm{d} \mathbf{X}(t)}{\mathrm{d} t}=\mathbf{F}(t) \mathbf{X}(t)+\mathbf{S}(t)
$$

or

$$
\frac{\mathrm{d} \mathbf{X}(t)}{\mathrm{d} t}=\left\{\mathbf{F}_{0}(t)+\epsilon \mathbf{F}_{1}(t)\right\} \mathbf{X}(t)+\mathbf{S}(t) .
$$

Here $\mathbf{F}_{0}(t)$ and $\mathbf{F}_{1}(t)$ are the sure and fluctuating part of $\mathbf{F}(t)$, respectively. We treat the fluctuating part of $F(t)$ as a perturbation of the sure part of the same. The parameter $\epsilon$ determines the size of the fluctuations. We assume that $\epsilon$ is small and the mean of the fluctuating part is zero, i.e.

$$
\left\langle F_{1}(t)\right\rangle=0 \text {. }
$$

The inhomogenous part of $\mathbf{S}(t)$ will be referred to as the source term.

Let the solution of the unperturbed homogenous equation be $\mathbf{Y}\left(t \mid t^{\prime}\right)$, defined by

$$
\frac{\mathrm{d} \mathbf{Y}\left(t \mid t^{\prime}\right)}{\mathrm{d} t}=\mathbf{F}_{0}(t) \mathbf{Y}\left(t \mid t^{\prime}\right) ; \quad \mathbf{Y}\left(t^{\prime} \mid t^{\prime}\right)=1
$$

A more explicit expression for the 'evolution matrix', $\mathbf{Y}(t)$, can be obtained by solving (33). The repeated iterations of the integration of the first and the use of the second yields

$$
\begin{aligned}
\mathbf{Y}(t \mid 0)=1 & +\int_{0}^{t} \mathrm{~d} t_{1} \mathbf{F}_{0}\left(t_{1}\right)+\int_{0}^{t} \mathrm{~d} t_{1} \int_{0}^{t_{1}} \mathrm{~d} t_{2} \\
& \times \mathbf{F}_{0}\left(t_{1}\right) \mathbf{F}_{0}\left(t_{2}\right)+\ldots ; \quad t_{n} \leq t_{n-1}
\end{aligned}
$$


One can extend the integrations making all of them from $t_{i}=0$ to $t_{i}=t(i=1,2, \ldots, n)$. To compensate for the larger integration domain a factor of $1 / n !$ is required

$$
\begin{aligned}
\mathbf{Y}(t \mid 0)= & \sum_{n=0}^{\infty} \frac{1}{n !} \int_{0}^{t} \mathrm{~d} t_{1} \int_{0}^{t} \mathrm{~d} t_{2} \ldots \int_{0}^{t} \mathrm{~d} t_{n} \\
& \times\left\lceil\mathbf{F}_{0}\left(t_{1}\right) \mathbf{F}_{0}\left(t_{2}\right) \ldots \mathbf{F}_{0}\left(t_{n}\right)\right\rceil \\
= & {\left[\exp \left\{\int_{0}^{t} \mathrm{~d} t^{\prime} \mathbf{F}_{0}\left(t^{\prime}\right)\right\}\right] . }
\end{aligned}
$$

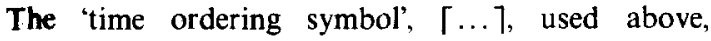
denotes that the operators be so rearranged that they appear in the decreasing order of their time arguments (after expanding the exponential in the second case). Now we introduce the interaction representations,

$$
\begin{gathered}
\mathbf{X}(t)=\mathbf{Y}(t \mid 0) \mathbf{v}(t) \\
\mathbf{F}_{1}(t)=\mathbf{Y}(t \mid 0) \mathbf{V}(t) \mathbf{Y}(0 \mid t)
\end{gathered}
$$

which transforms $(31 \mathrm{~b})$ into

$$
\begin{aligned}
\dot{\mathbf{Y}}(t \mid 0) \mathbf{v}(t)+\mathrm{Y}(t \mid 0) \dot{\mathbf{v}}(t) & \\
= & {\left[\mathbf{F}_{0}(t)+\epsilon \mathbf{F}_{1}(t)\right] \mathbf{Y}(t \mid 0) \mathbf{v}(t)+\mathbf{S}(t) . }
\end{aligned}
$$

Here, the first terms on both sides cancel due to (33). Multiplying by $\mathbf{Y}(0 \mid t)$ from the left one gets

$$
\dot{\mathbf{v}}(t)=\epsilon \mathbf{Y}(\mathbf{0} \mid t) \mathbf{F}_{1}(t) \mathbf{Y}(t \mid 0) \mathbf{v}(t)+\mathbf{Y}(0 \mid t) \mathbf{S}(t)
$$

Eliminating $\mathbf{F}_{1}(t)$ by $(36)$ yields

$$
\dot{\mathbf{v}}(t)=\epsilon \mathbf{V}(t) \mathbf{v}(t)+\mathbf{Y}(\mathbf{0} \mid t) \mathbf{S}(t) .
$$

Integrating (37) and iterating once, one gets

$$
\begin{aligned}
\mathbf{v}(t)= & \mathbf{v}(0)+\epsilon \int_{0}^{t} \mathrm{~d} t^{\prime} \mathbf{V}\left(t^{\prime}\right) \\
& \times\left\{\mathbf{v}(0)+\epsilon \int_{0}^{t^{\prime}} \mathrm{d} t^{\prime \prime} \mathbf{V}\left(t^{\prime \prime}\right) \mathbf{v}\left(t^{\prime \prime}\right)+\int_{0}^{t^{\prime}} \mathrm{d} t^{\prime \prime} \mathbf{Y}\left(0 \mid t^{\prime \prime}\right) \mathbf{S}\left(t^{\prime \prime}\right)\right\} \\
& +\int_{0}^{t} \mathrm{~d} t^{\prime} \mathbf{Y}\left(0 \mid t^{\prime}\right) \mathbf{S}\left(t^{\prime}\right) .
\end{aligned}
$$

One can average it and set $\langle\mathrm{V}(t)\rangle=0$ [cf. (32) and (36)].

$$
\begin{aligned}
\langle\mathbf{v}(t)\rangle= & \mathbf{v}(0)+\epsilon^{2} \int_{0}^{t} \mathrm{~d} t^{\prime} \int_{0}^{t^{\prime}} \mathrm{d} t^{\prime \prime}\left\langle\mathbf{V}\left(t^{\prime}\right) \mathbf{V}\left(t^{\prime \prime}\right) \mathbf{v}\left(t^{\prime \prime}\right)\right\rangle \\
& +\epsilon \int_{0}^{t} \mathrm{~d} t^{\prime} \int_{0}^{t^{\prime}} \mathrm{d} t^{\prime \prime}\left\langle\mathbf{V}\left(t^{\prime}\right) \mathbf{Y}\left(0 \mid t^{\prime \prime}\right) \mathbf{S}\left(t^{\prime \prime}\right)\right\rangle \\
& +\int_{0}^{t} \mathrm{~d} t^{\prime} \mathbf{Y}\left(0 \mid t^{\prime}\right)\left\langle\mathbf{S}\left(t^{\prime}\right)\right\rangle
\end{aligned}
$$

Now we make the following assumption which may be called 'Bourret's Approximation'.

$$
\left\langle\mathbf{V}\left(t^{\prime}\right) \mathbf{V}\left(t^{\prime \prime}\right) \mathbf{v}\left(t^{\prime \prime}\right)\right\rangle \simeq\left\langle\mathbf{V}\left(t^{\prime}\right) \mathbf{V}\left(t^{\prime \prime}\right)\right\rangle\left\langle\mathbf{v}\left(t^{\prime \prime}\right)\right\rangle
$$

Inserting (39) in (38) and then differentiating one gets,

$$
\begin{aligned}
\frac{\mathrm{d}}{\mathrm{d} t}\langle\mathbf{v}(t)\rangle= & \epsilon^{2} \int_{0}^{t} \mathrm{~d} t^{\prime}\left\langle\mathbf{V}(t) \mathbf{V}\left(t^{\prime}\right)\right\rangle\left\langle\mathbf{v}\left(t^{\prime}\right)\right\rangle \\
& +\epsilon \int_{0}^{t} \mathrm{~d} t^{\prime}\left\langle\mathbf{V}(t) \mathbf{Y}\left(0 \mid t^{\prime}\right) \mathbf{S}\left(t^{\prime}\right)\right\rangle+\mathbf{Y}(0 \mid t)\langle\mathbf{S}(t)\rangle
\end{aligned}
$$

In the original variables $\mathbf{X}$ and $\mathbf{F}_{1}$, the above equation reads as

$$
\begin{aligned}
\frac{\mathrm{d}}{\mathrm{d} t}\langle\mathbf{X}(t)\rangle= & \left\{\mathbf{F}_{0}+\epsilon^{2} \int_{0}^{t} \mathrm{~d} t^{\prime}\left\langle\mathbf{F}_{1}(t) \mathbf{Y}\left(t \mid t^{\prime}\right) \mathbf{F}_{1}\left(t^{\prime}\right)\right\rangle\right\}\langle\mathbf{X}(t)\rangle \\
& +\epsilon \int_{0}^{t} \mathrm{~d} t^{\prime}\left\langle\mathbf{F}_{1}(t) \mathbf{Y}\left(t \mid t^{\prime}\right) \mathbf{S}\left(t^{\prime}\right)\right\rangle+\langle\mathbf{S}(t)\rangle .
\end{aligned}
$$

Following Van Kampen we call the above equation 'Bourret's integral equation'.

Before proceeding further we try to justify 'Bourret's approximation', where an average of product has been replaced by a product of averages. We note that there are three time scales involved in (38). The scale on which $\mathbf{v}$ varies is the first one. If we take the order of $\mathbf{V}$ as unity, this time scale is measured by $1 / \epsilon$. The scale on which $\mathbf{V}$ varies is the next one but is not important for this discussion. The last one is the correlation time $\tau_{c}$ of $\mathbf{V}$. If this correlation time is very short, $\epsilon \tau_{c}$ is very small. Then one is able to divide the time axis in intervals $\Delta t$ satisfying

$$
\epsilon \tau_{c} \ll \epsilon \Delta t \ll 1 .
$$

During such a time interval $\Delta t, \mathrm{~V}$ forgets its past but $\mathbf{v}$ varies very little however. Thus in the first interval of the first integral of (38) $v\left(t^{\prime \prime}\right)$ can be approximated as

$$
\begin{aligned}
\mathbf{v}\left(t^{\prime \prime}\right) & =\mathbf{v}(0)+\epsilon \int_{0}^{\Delta t} \mathrm{~d} t^{\prime} \mathbf{V}\left(t^{\prime}\right) \mathbf{v}\left(t^{\prime}\right)+\int_{0}^{\Delta t} \mathrm{~d} t^{\prime \prime} \mathbf{Y}\left(0 \mid t^{\prime \prime}\right) \mathbf{S}\left(t^{\prime \prime}\right) \\
& \simeq \mathbf{v}(0) \simeq\left\langle\mathbf{v}\left(t^{\prime \prime}\right)\right\rangle
\end{aligned}
$$

where we have neglected the higher power of $\epsilon \Delta t$ and assumed the effect of source term in the integral would be negligible. Since the first integral of (38) is already of the order of $(\epsilon \Delta t)^{2}$, we are, in effect, neglecting terms of the order of $(\epsilon \Delta t)^{3}$. Of course the neglected source term is of the order of $(\epsilon \Delta t)^{2}$ and the assumption of a weak correlation between $\mathbf{S}(t)$ and $\mathbf{V}(t) \mathbf{V}\left(t^{\prime}\right)$ is necessary.

Thus integrating over the first interval we express $v(t+\Delta t)$ in terms of $v(t)$ and integrating over the second interval we can express $\mathbf{v}(t+2 \Delta t)$ in terms of $v(t+\Delta t)$ and so on. The all important point is that the values of $\mathbf{V}\left(t^{\prime}\right)$ during the two successive time intervals are practically uncorrelated. Thus in all intervals, one can use the same unbiased averages of 
$\mathbf{V}\left(t^{\prime}\right)$, instead of the averages conditioned by the knowledge of the evolution of $\mathbf{v}$ in the previous interval. So the Ansatz (39) is justified.

To obtain a differential equation instead of the integral equation (4l), we substitute $t^{\prime}=t-\tau$ in (40).

$$
\begin{aligned}
\frac{\mathrm{d}}{\mathrm{d} t}\langle\mathbf{v}(t)\rangle= & \epsilon^{2} \int_{0}^{t} \mathrm{~d} \tau\langle\mathbf{V}(t) \mathbf{V}(t-\tau)\rangle\langle\mathbf{v}(t-\tau)\rangle \\
& +\epsilon \int_{0}^{t} \mathrm{~d} \tau\langle\mathbf{V}(t) \mathbf{Y}(0 \mid t-\tau) \mathbf{S}(t-\tau)\rangle \\
& +\mathbf{Y}(0 \mid t)\langle\mathbf{S}(t)\rangle .
\end{aligned}
$$

Since we have already assumed that $\mathbf{V}(t)$ and $\mathbf{V}(t-\tau)$ are uncorrelated when $\tau>\tau_{c}$, we have $\langle\mathbf{V}(t) \mathbf{V}(t-\tau)\rangle$ $=\langle\mathbf{V}(t)\rangle\langle\mathbf{V}(t-\tau)\rangle=0 ; \tau\rangle \tau_{c}$ [cf. (32) and (36)]. Therefore whenever $t$ is greater than $\tau_{c}$, the first integral above vanishes and hence no error is made by extending the integral from $t$ to $\infty$. Assuming a weak correlation between $\mathbf{V}$ and $\mathbf{S}$ we can extend the second integral too. Therefore we get

$$
\begin{aligned}
\frac{\mathrm{d}}{\mathrm{d} t}\langle\mathbf{v}(t)\rangle \simeq \epsilon^{2} \int_{0}^{\infty} \mathrm{d} \tau\langle\mathbf{V}(t) \mathbf{V}(t-\tau)\rangle\langle\mathbf{v}(t)\rangle \\
+\epsilon \int_{0}^{\infty} \mathrm{d} \tau\langle\mathbf{V}(t) \mathbf{Y}(0 \mid t-\tau) \mathbf{S}(t-\tau)\rangle \\
+\mathbf{Y}(0 \mid t)\langle\mathbf{S}(t)\rangle .
\end{aligned}
$$

Here, besides extending the integral, we have also replaced $\langle\mathbf{v}(t-\tau)\rangle$ by $\langle\mathbf{v}(t)\rangle$. Since the first integral is almost solely contributed by the time $\left(0, \tau_{c}\right)$, the relative error due to this replacement is of the order of $\tau_{c}\langle\dot{v}\rangle /\langle\mathbf{v}\rangle$. Because we have assumed $\mathbf{V}(t)$ is of the order of unity, by (44) itself $\langle\dot{\boldsymbol{v}}\rangle \approx \epsilon^{2} \tau_{c}\langle\mathbf{v}\rangle$ where we have neglected the effect of the source again. The relative error is $\left(\epsilon \tau_{c}\right)^{2}$ and we have already neglected terms of relative order $\epsilon \tau_{c}$. So the error due to the replacement can be ignored. (44) can be easily transformed to the original representations of $\mathbf{X}, \mathbf{F}_{0}$ and $\mathbf{F}_{1}$. Since in all our applications $\mathbf{F}_{0}$ will be independent of time, we write the transformed equation specifically for a constant $\mathbf{F}_{0}$, i.e. $\mathbf{Y}(t \mid 0)=\exp \left(\mathbf{F}_{0} t\right)$.

$$
\begin{aligned}
\frac{\mathrm{d}}{\mathrm{d} t}\langle\mathbf{X}(t)\rangle= & \left\{\mathbf{F}_{0}+\epsilon^{2} \int_{0}^{\infty} \mathrm{d} \tau\left\langle\mathbf{F}_{1}(t) \mathrm{e}^{\mathbf{F}_{0} \tau} \mathbf{F}_{1}(t-\tau)\right\rangle \mathrm{e}^{-\mathbf{F}_{0} \tau}\right\} \\
& \times\langle\mathbf{X}(t)\rangle+\epsilon \int_{0}^{\infty} \mathrm{d} \tau\left\langle\mathbf{F}_{1}(t) \mathrm{e}^{\mathbf{F}_{0} r} \mathrm{~S}(t-\tau)\right\rangle \\
& +\langle\mathbf{S}(t)\rangle .
\end{aligned}
$$

We can apply (45) not only to determine the mean but also the variance and power spectral density of $\mathbf{X}$, by manipulating the original equation as follows:

$$
\frac{\mathrm{d} \mathbf{X}}{\mathrm{d} t}=\mathbf{F X}+\mathbf{S}
$$

Taking transpose, we get

$$
\frac{\mathrm{d} \mathbf{X}^{T}}{\mathrm{~d} t}=\mathbf{X}^{T} \mathbf{F}^{T}+\mathbf{S}^{T} .
$$

Multiplying (31a) by $\mathbf{X}^{T}$ from the right and (46) from the left and adding the resulting equations we get

$$
\frac{\mathrm{d}}{\mathrm{d} t}\left(\mathbf{X} \mathbf{X}^{T}\right)=\mathbf{F}\left(\mathbf{X} \mathbf{X}^{T}\right)+\left(\mathbf{X} \mathbf{X}^{T}\right) \mathbf{F}^{T}+\left(\mathbf{S} \mathbf{X}^{T}+\mathbf{X} \mathbf{S}^{T}\right) .
$$

The components of the square matrix $\mathbf{X X}^{r}$ can be rearranged in a column matrix so that an equation of the form (3la) is obtained. We show the results for a two-component $\mathbf{X}$

$$
\begin{aligned}
\frac{\mathrm{d}}{\mathrm{d} t}\left[\begin{array}{c}
X_{1}^{2} \\
X_{1} X_{2} \\
X_{2}^{2}
\end{array}\right]= & {\left[\begin{array}{ccc}
2 f_{11} & 2 f_{12} & 0 \\
f_{21} & f_{11}+f_{21} & f_{12} \\
0 & 2 f_{21} & 2 f_{22}
\end{array}\right]\left[\begin{array}{c}
X_{1}^{2} \\
X_{1} X_{2} \\
X_{2}^{2}
\end{array}\right] } \\
& +\left[\begin{array}{c}
2 S_{1} X_{1} \\
S_{1} X_{1}+S_{2} X_{2} \\
2 S_{1} X_{2}
\end{array}\right] .
\end{aligned}
$$

One can apply (45) to equation (47) to get the differential equations for $\left\langle X_{1}^{2}\right\rangle,\left\langle X_{1} X_{2}\right\rangle$ etc. from which variance can be easily obtained. Similarly, to obtain the correlation matrix (and then power spectral density), we simply multiply (31a) by $\mathbf{X}^{T}\left(t_{0}\right)$ to get

$$
\frac{\mathrm{d}}{\mathrm{d} t}\left[\mathbf{X}(t) \mathbf{X}^{T}\left(t_{0}\right)\right]=\mathbf{F}\left[\mathbf{X}(t) \mathbf{X}^{T}\left(t_{0}\right)\right]+\mathbf{S}(t) \mathbf{X}^{T}\left(t_{0}\right) ; \quad t_{0}<t
$$

which may be split into two equations as

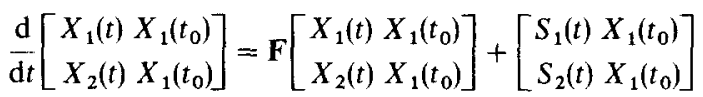
and

$\frac{\mathrm{d}}{\mathrm{d} t}\left[\begin{array}{cc}X_{1}(t) & X_{2}\left(t_{0}\right) \\ X_{2}(t) & X_{2}\left(t_{0}\right)\end{array}\right]=\mathbf{F}\left[\begin{array}{ll}X_{1}(t) & X_{2}\left(t_{0}\right) \\ X_{2}(t) & X_{2}\left(t_{0}\right)\end{array}\right]+\left[\begin{array}{ll}S_{1}(t) & X_{2}\left(t_{0}\right) \\ S_{2}(t) & X_{2}\left(t_{0}\right)\end{array}\right]$.

One may again apply (45) to the above two equations and combine them to get the equation for correlation matrix of $\mathbf{X}$ as follows:

$$
\begin{aligned}
\frac{\mathrm{d}}{\mathrm{d} t}\left\langle\mathbf{X}(t) \mathbf{X}^{T}\left(t_{0}\right)\right\rangle \\
=\left\{\mathbf{F}_{0}+\epsilon^{2} \int_{0}^{\infty} \mathrm{d} \tau\left\langle\mathbf{F}_{1}(t) \mathrm{e}^{\mathbf{F}_{0} \tau} \mathbf{F}_{1}(t-\tau)\right\rangle \mathrm{e}^{-\mathbf{F}_{0} \tau}\right\} \\
\quad \times\left\langle\mathbf{X}(t) \mathbf{X}^{T}\left(t_{0}\right)\right\rangle+\epsilon \int_{0}^{\infty} \mathrm{d} \tau \\
\quad \times\left\langle\mathbf{F}_{1}(t) \mathrm{e}^{\mathbf{F}_{0} \tau} \mathbf{S}(t-\tau) \mathbf{X}^{T}\left(t_{0}\right)\right\rangle+\left\langle\mathbf{S}(t) \mathbf{X}^{T}\left(t_{0}\right)\right\rangle .
\end{aligned}
$$

It may be noted here that in such manipulation of the original equation ( $31 \mathrm{a})$, the largest value of the correlation time $\tau_{c}$ for which (45) remains valid may change.

Now we apply these results on the PRKE to obtain the following statistical properties of reactor power and delayed neutron precursor density. 


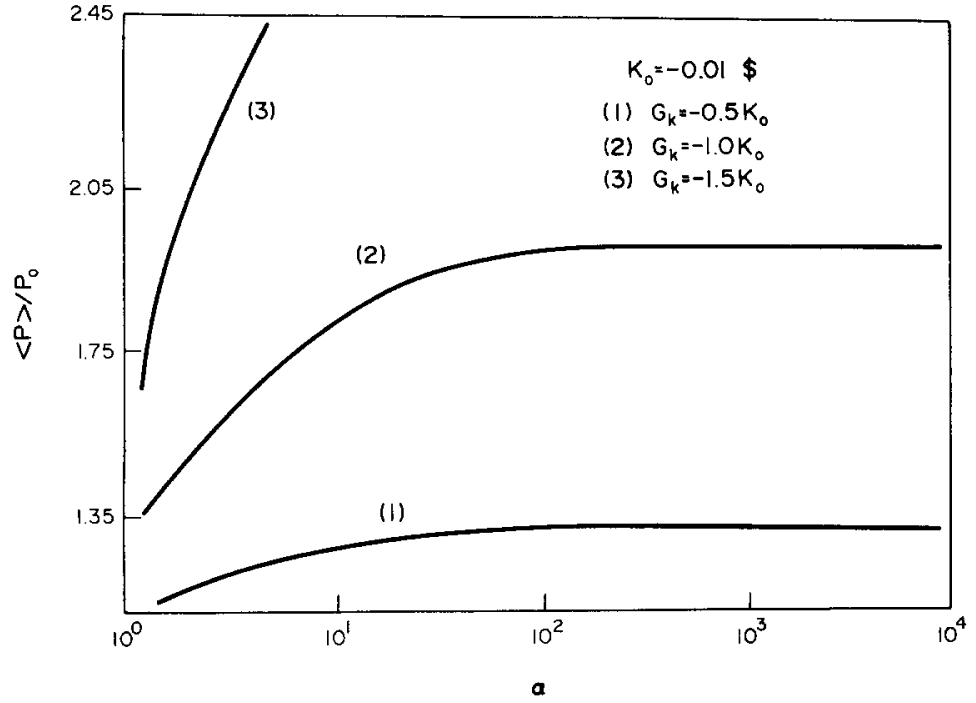

Fig. 1. Normalized mean power $\langle P\rangle / P_{0}$, as a function of inverse correlation time, $\alpha$.

\subsection{The behavior of the mean}

We shall discuss two cases--neglecting and considering the delayed neutrons.

(i) Neglecting the delayed neutrons. The PRKE in this case is given by

$$
\frac{\mathrm{d} P(t)}{\mathrm{d} t}=\left\{K_{0}+k(t)\right\} P(t)+S_{0}
$$

and the application of (45) is straightforward. One obtains,

$$
\begin{aligned}
\frac{\mathrm{d}}{\mathrm{d} t}\langle P(t)\rangle= & \left\{K_{0}+\int_{0}^{\infty} \mathrm{d} \tau\right. \\
& \left.\times\left\langle k(t) \mathrm{e}^{K_{0} \tau} k(t-\tau)\right\rangle \mathrm{e}^{-K_{0} \tau}\right\}\langle P(t)\rangle+S_{0} \\
= & \left\{K_{0}+\int_{0}^{\infty} \mathrm{d} \tau \phi_{k}(\tau)\right\}\langle P(t)\rangle+S_{0} .
\end{aligned}
$$

The second integral of (45) vanishes because of $\langle k(t) S(t-\tau)\rangle=\langle k(t)\rangle S_{0}=0$. As an example we consider a non-white reactivity noise whose autocorrelation function is given by

$$
\phi_{k}(\tau)=\frac{1}{2} G_{k} \alpha \mathrm{e}^{-\alpha|\tau|} .
$$

Inserting (51) in (50) we get

$$
\frac{\mathrm{d}}{\mathrm{d} t}\langle P(t)\rangle=\left(K_{0}+\frac{1}{2} G_{k}\right)\langle P(t)\rangle+S_{0}
$$

where we have assumed that $|\alpha|>K_{0}$. At stationary state,

$$
\langle P\rangle=-S_{0} /\left(K_{0}+\frac{1}{2} G_{k}\right) ; \quad K_{0}+\frac{1}{2} G_{k}<0 .
$$

This is the same as the results obtained using the Fokker-Planck theory as well as averaging directly, by Akcasu and Karasulu (1976).

(ii) Considering the delayed neutrons. In this case the PRKE can be written as

$$
\frac{\mathrm{d} \mathbf{X}}{\mathrm{d} t}=\{\mathbf{M}+\alpha(t)\} \mathbf{X}(t)+\mathbf{S}_{0},
$$

where

$$
\mathbf{X}=\left[\begin{array}{l}
P \\
C
\end{array}\right] ; \quad \mathbf{M}=\left[\begin{array}{lr}
K_{0}-1 & \lambda \\
1 & -\lambda
\end{array}\right] ; \quad \mathbf{S}_{0}=\left[\begin{array}{c}
S_{0} \\
0
\end{array}\right]
$$

and

$$
\alpha(t)=k(t) \mathbf{A} ; \quad \mathbf{A}=\left[\begin{array}{ll}
1 & 0 \\
0 & 0
\end{array}\right] .
$$

Comparing (53) with (31b) and using (45), one obtains

$$
\begin{aligned}
\frac{\mathrm{d}}{\mathrm{d} t}\langle\mathbf{X}(t)\rangle= & \left\{\mathbf{M}+\int_{0}^{\infty} \mathrm{d} \tau\left\langle\alpha(t) \mathrm{e}^{\mathbf{M} \tau} \alpha(t-\tau)\right\rangle \mathrm{e}^{-\mathbf{M r}}\right\} \\
& \times\langle\mathbf{X}(t)\rangle+\int_{0}^{\infty} \mathrm{d} \tau\left\langle\boldsymbol{\alpha}(t) \mathrm{e}^{\mathbf{M r}} \mathbf{S}_{0}\right\rangle+\left\langle\mathbf{S}_{0}\right\rangle .
\end{aligned}
$$

As in case (i), the second integral is zero due to $\langle\alpha(t)\rangle=0$. The integrand of the first integral may be written as $\langle k(t) k(t-\tau)\rangle \mathbf{A} \exp (\mathbf{M} \tau) \mathbf{A} \exp (-\mathbf{M} \tau)$. We note that the eigenvalues of $\mathbf{M}$ are given by $\gamma_{1}$ and $\gamma_{2}$ [cf. (10) and (11)]. The eigenvectors are col[ $\left.\lambda+\gamma_{1} 1\right]$ and $\operatorname{col}\left[\begin{array}{ll}\lambda+\gamma_{2} & 1\end{array}\right]$, and the diagonalizing matrices are

$$
\begin{aligned}
\mathbf{R} & =\left[\begin{array}{cc}
\lambda+\gamma_{1} & \lambda+\gamma_{2} \\
1 & 1
\end{array}\right] ; \\
\mathbf{R}^{-1} & =\left(\gamma_{1}-\gamma_{2}\right)^{-1}\left[\begin{array}{cc}
1 & -\left(\lambda+\gamma_{2}\right) \\
-1 & \lambda+\gamma_{1}
\end{array}\right] .
\end{aligned}
$$


Using the results

$$
\mathbf{R}^{-1} \mathbf{e}^{\mp \mathbf{M} \tau} \mathbf{R}=\left[\begin{array}{lr}
\mathbf{e}^{\mp \gamma_{1} \tau} & 0 \\
0 & \mathbf{e}^{\mp \gamma_{2 \tau} \tau}
\end{array}\right],
$$

(55) reduces to

$$
\begin{aligned}
\frac{\mathrm{d}}{\mathrm{d} t}\left[\begin{array}{c}
\langle P\rangle \\
\langle C\rangle
\end{array}\right]= & \left\{\mathbf{M}+\int_{0}^{\infty} \mathrm{d} \tau \phi_{\boldsymbol{k}}(\tau)\left(\gamma_{1}-\gamma_{2}\right)^{-2} \boldsymbol{W}\right\} \\
& \times\left[\begin{array}{c}
\langle P\rangle \\
\langle C\rangle
\end{array}\right]+\left[\begin{array}{c}
S_{0} \\
0
\end{array}\right]
\end{aligned}
$$

where

$$
\begin{aligned}
& W_{11}=a_{1}^{2}-a_{1} a_{2}\left(\mathrm{e}^{a_{0} t}+\mathrm{e}^{-a_{0} \tau}\right)+a_{2}^{2} \\
& W_{12}=a_{1} a_{2}\left[a_{1} \mathrm{e}^{a_{0} \tau}+a_{2} \mathrm{e}^{-a_{0} \tau}-\left(a_{1}+a_{2}\right)\right] \\
& W_{21}=W_{22}=0
\end{aligned}
$$

and

$$
a_{0}=\gamma_{1}-\gamma_{2} ; \quad a_{1}=\lambda+\gamma_{1} ; \quad a_{2}=\lambda+\gamma_{2} .
$$

We again consider the same non-white reactivity, whose autocorrelation function is given by (51) as an example. Inserting (51) in (58) we get

$$
\begin{aligned}
\frac{\mathrm{d}}{\mathrm{d} t}\left[\begin{array}{c}
\langle P\rangle \\
\langle C\rangle
\end{array}\right]= & \left\{\mathbf{M}+\frac{1}{2} G_{k}\left[\begin{array}{cc}
I_{1} & I_{2} \\
0 & 0
\end{array}\right]\right\} \\
& \times\left[\begin{array}{c}
\langle P\rangle \\
\langle C\rangle
\end{array}\right]+\left[\begin{array}{c}
S_{0} \\
0
\end{array}\right],
\end{aligned}
$$

where

$$
\begin{gathered}
I_{1}=\frac{1}{a_{0}^{2}}\left\{a_{1}^{2}+a_{2}^{2}-2 a_{1} a_{2} \alpha^{2} /\left(\alpha^{2}-a_{0}^{2}\right)\right\} \\
I_{2}=\frac{a_{1} a_{2}}{a_{0}^{2}}\left\{-\left(a_{1}+a_{2}\right)+a_{1} \alpha /\left(\alpha-a_{0}\right)+a_{2} \alpha /\left(\alpha+a_{0}\right)\right\} .
\end{gathered}
$$

In the above it was assumed that

$$
\alpha>\left|a_{0}\right|=\left[\left(\lambda-K_{0}+1\right)^{2}+4 \lambda K_{0}\right]^{\frac{1}{2}} .
$$

When this condition is not satisfied, the integrals above diverge. $\alpha$ is, in fact, the inverse of correlation time. While deriving Bourret's integral equation (38), short correlation time was assumed, i.e. $1 / \alpha$ is small compared to the time scale on which the deterministic equation evolves. In this case that time scale is unity $(\ell / \beta)$. Since $\lambda \ll 1$, near criticality, $\left|a_{0}\right| \approx \lambda-K_{0}$ $+1 \approx 1$ and thus we see that Bourret's approximation breaks down when the correlation time is about unity or greater.

At stationary state we find the mean values as

$$
\begin{gathered}
\langle P\rangle=-S_{0} /\left(K_{0}+\frac{1}{2} G_{k} \beta\right) \\
\langle C\rangle=\langle P\rangle / \lambda,
\end{gathered}
$$

where

$$
\beta=I_{1}+I_{2} / \lambda .
$$

A physically realizable power exists only when $K_{0}+\frac{1}{2} G_{k} \beta<0$. The effect of the correlation time on the reactor power can be seen graphically in Fig. 1. We observe that noise increases mean power and does it more when the correlation time is shorter. Mean power is maximum when the correlation time is zero, i.e. when the noise is white. As $\alpha \rightarrow \infty$ the reactivity noise becomes white and $I_{1}$ and $I_{2}$ reduce to 1 and 0 , respectively, and $\beta$ becomes 1 . Using these values of $\beta$ in (63a) we obtain results identical to those obtained by Akcasu and Karasulu (1976) using the Fokker-Planck theory.

\subsection{The variance of the reactor power}

Applying (47) on (53) we get

$$
\frac{\mathrm{d}}{\mathrm{d} t} \mathbf{Y}(t)=\left\{\mathbf{A}_{0}+k(t) \mathbf{A}_{1}\right\} \mathbf{Y}(t)+\mathbf{S}_{\boldsymbol{y}},
$$

where

$$
\begin{aligned}
\mathbf{Y} & =\operatorname{col}\left[\begin{array}{lll}
P^{2} & P C & C^{2}
\end{array}\right] \\
\mathbf{S}_{y} & =\operatorname{col}\left[\begin{array}{lll}
2 S_{0} P & S_{0} C & 0
\end{array}\right]
\end{aligned}
$$

and

$$
\mathbf{A}_{0}=\left[\begin{array}{ccc}
2\left(K_{0}-1\right) & 2 \lambda & 0 \\
1 & K_{0}-1-\lambda & \lambda \\
0 & 2 & -2 \lambda
\end{array}\right]
$$

$$
\mathbf{A}_{1}=\left[\begin{array}{lll}
2 & 0 & 0 \\
0 & 1 & 0 \\
0 & 0 & 0
\end{array}\right] .
$$

Using (48) we obtain

$$
\begin{aligned}
\frac{\mathrm{d}}{\mathrm{d} t}\langle\mathbf{Y}(t)\rangle= & \left\{\mathbf{A}_{0}+\int_{0}^{\infty} \mathrm{d} \tau\right. \\
& \left.\times\langle k(t) k(t-\tau)\rangle \mathbf{A}_{1} \mathrm{e}^{\mathbf{A}_{0} \tau} \mathbf{A}_{1} \mathrm{e}^{-\mathbf{A}_{0} \tau}\right\}\langle\mathbf{Y}(t)\rangle \\
& +\int_{0}^{\infty} \mathrm{d} \tau \mathbf{A}_{1} \mathrm{e}^{\mathbf{A}_{0} \tau}\left\langle k(t) \mathbf{S}_{\mathbf{y}}(t-\tau)\right\rangle+\left\langle\mathbf{S}_{y}\right\rangle
\end{aligned}
$$

Since we are not considering any feedback, by causality, the correlations $\langle k(t) P(t-\tau)\rangle$ and $\langle k(t) C(t-\tau)\rangle$ are zero and therefore the second integral vanishes. As before, the integrand of the first integral can be found by determining the eigenvalues $\delta_{1}$, $\delta_{2}, \delta_{3}$ and the matrix formed by the eigenvectors, $\mathbf{S}$, of $\mathbf{A}_{\mathbf{0}}$.

$$
\delta_{1}=\left(K_{0}-1-\lambda\right) ; \delta_{2 ; 3}=\delta_{1} \mp\left(\delta_{1}^{2}+4 \lambda K_{0}\right)^{\frac{1}{2}}
$$


$S_{1 j}=2 \lambda\left(2 \lambda+\delta_{j}\right) ; \quad S_{2 j}=\left(2 \lambda+\delta_{j}\right)\left(\delta_{j}-2 K_{0}+2\right)$

$$
S_{3 j}=2\left(\delta_{j}-2 K_{0}+2\right) \text {. }
$$

The first integral of (65) reads as

$$
\int_{0}^{\infty} \mathrm{d} \tau \phi_{k}(\tau) \sum_{m, n} \mathrm{e}^{\left(\delta_{m}-\delta_{n}\right) \tau} \mathbf{D}_{m n}
$$

where

$$
\mathrm{D}_{m n}=\mathrm{C}_{m} \mathrm{C}_{n}
$$

and the elements of the matrix $\mathbf{C}_{m}$ are given by

$$
\left(C_{m}\right)_{i j}=\left(A_{1} S\right)_{i m} S_{m j}^{-1} .
$$

No summation is implied by repeated indices and it should be noted that $\mathbf{C}_{m} \neq\left(\mathbf{A}_{1} \mathbf{S}\right) \mathbf{S}^{-1}$.

Again we use the same reactivity correlation function [cf. (51)] as an example, and obtain by what goes above,

$$
\frac{\mathrm{d}}{\mathrm{d} t}\langle\mathbf{Y}(t)\rangle=\left(\mathbf{A}_{0}+\mathbf{B}\right)\langle\mathbf{Y}(t)\rangle+\left\langle\mathbf{S}_{\boldsymbol{y}}\right\rangle
$$

where

$$
B_{m n}=\frac{1}{2} G_{k} \sum_{m, n} D_{m n}\left[1-\left(\delta_{m}-\delta_{n}\right) / \alpha\right]^{-1} .
$$

Here we have considered $\alpha$ to be greater than the largest of the differences between any two eigenvalues, i.e. $\left(\delta_{m}-\delta_{n}\right)_{\max }$. Near criticality, i.e. $K_{0} \ll 1$, this means

$$
\alpha>\left|2\left[\left(1+\lambda-K_{0}\right)^{2}+4 \lambda K_{0}\right]^{\frac{1}{2}}\right| \approx 2 .
$$

The integral (67a) diverges if this condition is not satisfied. We note that the value of $\alpha$ at which Bourret's approximation breaks down in the computation of second moments is double of the same in the computation of the mean.

The differential equation for variance can be obtained easily from (68) and (60). Especially at the stationary state.

$$
\begin{aligned}
& {\left[C_{p p}(0) C_{p c}(0) C_{c c}(0)\right]^{T}=} \\
& \quad-\left(\mathbf{A}_{0}+\mathbf{B}\right)^{-1}\left\{( \mathbf { B } - \mathbf { H } ) \left[1^{\left.\left.1 / \lambda^{1} / \lambda^{2}\right]\right\}}\right.\right.
\end{aligned}
$$

where

$$
\mathbf{H}=\left[\begin{array}{ccc}
G_{k} I_{1} & G_{k} I_{2} & 0 \\
0 & \frac{1}{2} G_{k} I_{1} & \frac{1}{2} G_{k} I_{2} \\
0 & 0 & 0
\end{array}\right]
$$

and $I_{1}$ and $I_{2}$ are given by (61a) and (61b), respectively. It may be noted that the analytic evaluation of the matrix $B$ is laborious but the numerical evaluation is simple and straightforward. In the case of white noise reactivity insertion one lets $\alpha \rightarrow \infty$ in (69) or can evaluate the first integral of (65) directly as

$$
\begin{aligned}
\mathbf{B} & =\int_{0}^{\infty} \mathrm{d} \tau G_{k} \delta(\tau) \mathbf{A}_{1} \mathrm{e}^{\mathbf{A}_{0} \tau} \mathbf{A}_{1} \mathrm{e}^{-\mathbf{A}_{0} \tau} \\
& =\frac{1}{2} G_{k} \mathbf{A}_{1} \mathbf{A}_{1}=\frac{1}{2} G_{k}\left[\begin{array}{lll}
4 & 0 & 0 \\
0 & 1 & 0 \\
0 & 0 & 0
\end{array}\right] .
\end{aligned}
$$

Substituting $\left.\left\langle k(t) S_{y}(t-\tau)\right\rangle=0, \tau\right\rangle 0$ and (71) in (65) one gets

$$
\begin{aligned}
\frac{\mathrm{d}}{\mathrm{d} t}\left[\begin{array}{c}
\left\langle P^{2}\right\rangle \\
\langle P C\rangle \\
\left\langle C^{2}\right\rangle
\end{array}\right]= & {\left[\begin{array}{ccc}
2\left(K_{0}+\frac{1}{2} G_{k}-1\right) & 2 \lambda & 0 \\
1 & K_{0}-1-\lambda+\frac{1}{2} G_{k} & \lambda \\
0 & 2 & -2 \lambda
\end{array}\right] } \\
& \times\left[\begin{array}{c}
\left\langle P^{2}\right\rangle \\
\langle P C\rangle \\
\left\langle C^{2}\right\rangle
\end{array}\right]+S_{0}\left[\begin{array}{c}
2\langle P\rangle \\
\langle C\rangle \\
0
\end{array}\right]
\end{aligned}
$$

This result exactly corresponds to the result obtained by Akcasu and Karasulu (1976) using the FokkerPlanck theory. At stationary state (72) yields

$$
\frac{\left\langle P^{2}\right\rangle}{\langle P\rangle^{2}}=\frac{1}{2} G_{k}\left(\frac{K_{0}+\frac{1}{2} G_{k}}{K_{0}+\frac{1}{2} G_{k}-\lambda}-K_{0}-G_{k}\right)^{-1}
$$

$\langle P C\rangle=\langle C P\rangle=\lambda\left\langle C^{2}\right\rangle=\left\langle P^{2}\right\rangle /\left(\lambda-K_{0}-\frac{1}{2} G_{k}\right)$.

The dependence of variance to mean-squared ratio of reactor power on reactivity correlation time was obtained numerically from (70) and is shown here graphically in Fig. 2. We observe that the reactivity noise with larger magnitude and shorter correlation time increases the variance to mean-squared ratio more.

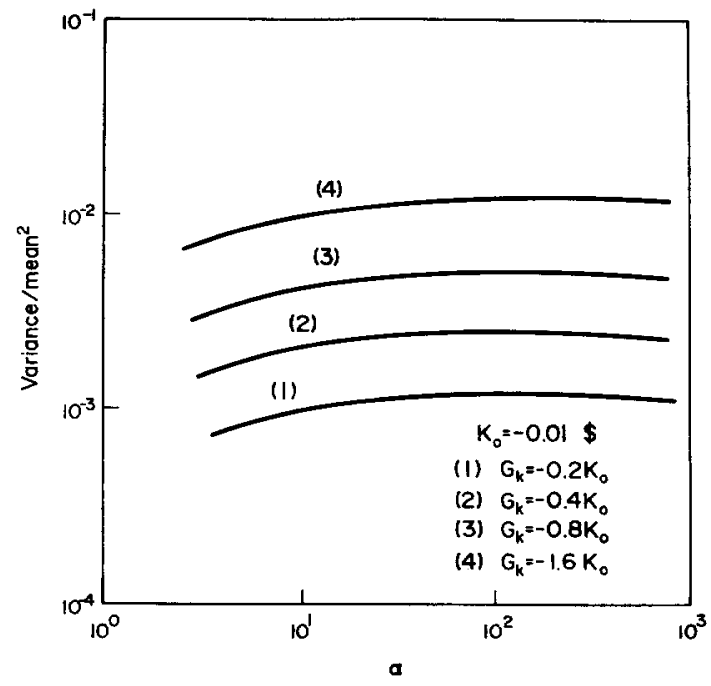

Fig. 2. Variance to mean-squared ratio as a function of inverse correlation time, $\alpha$ 


\subsection{The power spectral density}

In a similar fashion one can obtain two time correlation function by applying (53) on (48):

$$
\begin{aligned}
\frac{\mathrm{d}}{\mathrm{d} t}\langle & \left\langle\left[\begin{array}{l}
P(t) \\
C(t)
\end{array}\right]\left[P\left(t_{0}\right) C\left(t_{0}\right)\right]\right\rangle \\
= & \left\{\mathbf{M}+\int_{0}^{\infty} \mathrm{d} \tau \phi_{k}(\tau) \mathbf{A} \mathrm{e}^{\mathbf{M} \tau} \mathbf{A} \mathrm{e}^{-\mathbf{M}_{\tau}}\right\} \\
& \times\left\langle\left[\begin{array}{c}
P(t) \\
C(t)
\end{array}\right]\left[P\left(t_{0}\right) C\left(t_{0}\right)\right]\right\rangle \\
& +\left\langle\left[\begin{array}{c}
S_{0} \\
0
\end{array}\right]\left[P\left(t_{0}\right) C\left(t_{0}\right)\right]\right\rangle .
\end{aligned}
$$

Here we have used causality again. From (74) and (60) one can obtain the equation for covariance matrix. For the correlation function (51) we are using as an example (assuming $\alpha>2$ ), the stationary state results are given below.

$$
\frac{\mathrm{d}}{\mathrm{d} \tau} \mathbf{C}(\tau)=\mathbf{T C}(\tau), \quad \tau>\mathbf{0},
$$

where

$$
\mathbf{C}(\tau)=\left[\begin{array}{ll}
C_{p p}(\tau) & C_{p c}(\tau) \\
C_{c p}(\tau) & C_{c c}(\tau)
\end{array}\right]
$$

and

$$
\mathbf{T}=\mathbf{M}+\frac{1}{2} G_{k}\left[\begin{array}{cc}
I_{1} & I_{2} \\
0 & 0
\end{array}\right]
$$

The values of $\mathbf{C}(\tau)$ for $\tau<0$ is obtained from the relation $\mathbf{C}(-\tau)=\mathbf{C}^{T}(\tau)$. Taking the Laplace transform of (75a) and using the relation $\mathbf{G}(\omega)=\mathbf{C}(i \omega)+\mathbf{C}^{T}(i \omega)$ $=2 \operatorname{Re} C(i \omega)$, we get

$$
\mathbf{G}(\omega)=2 \operatorname{Re}\left\{(i \omega \mathbf{I}-\mathbf{T})^{-1} \mathbf{C}(0)\right\} .
$$

Especially, the power spectral density of power

$$
\begin{aligned}
& G_{p p}(\omega)=G_{11}(\omega) \text { is given explicitly as } \\
& \frac{G_{p p}(\omega)}{C_{p p}(0)} \\
& \quad=2 \operatorname{Re}\left\{\frac{(i \omega+\lambda)+\left(\lambda+\frac{1}{2} G_{k} I_{2}\right) C_{p c}(0) / C_{p p}(0)}{(i \omega+\lambda)\left(i \omega-K_{0}+1-\frac{1}{2} G_{k} I_{1}\right)-\left(\lambda+\frac{1}{2} G_{k} I_{2}\right)}\right\} \\
& \quad=\frac{2 \mathscr{K}\left|\left(i \omega+\omega_{3}\right)\right|^{2}}{\left|\left(i \omega+\omega_{1}\right)\right|^{2}\left|\left(i \omega+\omega_{2}\right)\right|^{2}},
\end{aligned}
$$

where the corner frequencies $\omega_{1}$ and $\omega_{2}$ are given by

$$
\begin{aligned}
= & \frac{1}{2}\left\{\left(\lambda-K_{0}+1-\frac{1}{2} G_{k} I_{1}\right)\right. \\
& \mp\left[\left(\lambda-K_{0}+1-\frac{1}{2} G_{k} I_{1}\right)^{2}\right. \\
& \left.\left.-4 \lambda\left(-K_{0}-\frac{1}{2} G_{k} I_{1}-G_{k} I_{2} / 2 \lambda\right)\right]^{1 / 2}\right\}
\end{aligned}
$$

and the corner frequency $\omega_{3}$ by

$$
\begin{aligned}
= & \mathscr{X}^{1 / 2}\left\{-\lambda\left(K_{0}+\frac{1}{2} G_{k} I_{1}+\frac{1}{2 \lambda} G_{k} I_{2}\right)\right. \\
& \left.\times\left(\lambda+\left[\lambda+\frac{1}{2} G_{k} I_{2}\right]\right) \frac{C_{p c}(0)}{C_{p p}(0)}\right\}^{1 / 2},
\end{aligned}
$$

\begin{tabular}{|c|c|c|c|c|}
\hline \multirow[b]{2}{*}{ Method } & \multirow{2}{*}{$\begin{array}{c}\text { Source } \\
\text { characteristic }\end{array}$} & \multicolumn{2}{|c|}{ Reactivity characteristic } & \multirow[b]{2}{*}{ Accuracy* } \\
\hline & & Distribution & Spectrum & \\
\hline $\begin{array}{l}\text { Conventional } \\
\text { linearization }\end{array}$ & $\begin{array}{l}\text { No } \\
\text { restriction }\end{array}$ & $\begin{array}{l}\text { No } \\
\text { restriction }\end{array}$ & $\begin{array}{l}\text { No } \\
\text { restriction }\end{array}$ & $\begin{array}{l}\text { Poor; effect of } G_{k} \text { on } \\
\langle P\rangle \text { is lost completely }\end{array}$ \\
\hline $\begin{array}{l}\text { Logarithmic } \\
\text { linearization }\end{array}$ & Constant & Gaussian & $\begin{array}{l}\text { No } \\
\text { restriction }\end{array}$ & $\begin{array}{l}\text { Fails to yield } \\
\text { exact result }\end{array}$ \\
\hline $\begin{array}{l}\text { Bourret's } \\
\text { approximation }\end{array}$ & $\begin{array}{l}\text { Constant; only mean } \\
\text { can be determined if } \\
\text { fiuctuating with a } \\
\text { short correlation time }\end{array}$ & $\begin{array}{l}\text { No } \\
\text { restriction }\end{array}$ & $\begin{array}{l}\text { Short } \\
\text { correlation } \\
\text { time }\end{array}$ & $\begin{array}{l}\text { Yields } \\
\text { exact result }\end{array}$ \\
\hline $\begin{array}{l}\text { Fokker-Planck } \\
\text { theory }\end{array}$ & $\begin{array}{l}\text { Gaussian, white; may } \\
\text { be correlated to } k(t) \\
\text { reactivity }\end{array}$ & Gaussian & White & Exact \\
\hline
\end{tabular}

where

$$
\mathscr{K}=1-\left(K_{0}+\frac{1}{2} G_{k} I_{1}\right)-\left(\lambda+\frac{1}{2} G_{k} I_{2}\right) C_{p c}(0) / C_{p p}(0) .
$$

Numerical evaluation of (77) is simple, once the variance is computed. Using similar numerical values as in Fig. 2, it was found that the reactivity correlation time $1 / \alpha$ has almost no effect on the power spectral density. The reason follows. Because Bourret's approximation fails when $\alpha<2$, we had to consider only those reactivity inputs which have cut-off frequencies higher than 2; whereas the resonances of subcritical reactor transfer function [cf. (10) and (11)] fall within about 1 . So the reactor 'sees' all such reactivity inputs essentially as white noise and therefore no dependency of power spectral density on correla-

Table 1. Comparison among the available methods for solving the Stochastic Point Reactor Kinetic Equations

* For the comparison of accuracy we have assumed $G_{k}$ to be large. Reactivity is also assumed to be white because only in that case all the methods compared above are applicable. 
tion time could be observed. Here the assumption $\alpha>2$ was necessary to find the variance. If one wants to find the two time correlation only then the assumption $\alpha>1$ is enough. In this case too, one may reproduce the exact results (cf. Akcasu and Karasulu, 1976) when the reactivity noise is white.

\section{COMPARISON, DISCUSSION AND CONCLUSION}

For the purpose of comparison we consider the reactivity noise to be Gaussian white, autocorrelation being given by $\phi_{k}(\tau)=G_{k} \delta(\tau)$. In this case the exact results have been obtained by Akcasu and Karasulu (1976) using the Fokker-Planck theory; and all the results obtained by Bourret's approximation reduce to these exact results. It can also be shown in general, that in the case of homogenous equation with Gaussian white noise, Bourret's approximation and the Fokker-Planck theory yield identical results.

However, the logarithmic linearization results do not coincide with the exact results. For example, if (15) is satisfied with $m=1$, the stationary values of mean reactor power are obtained as

$$
\langle P\rangle=P_{0} \exp \left\{\frac{G_{k}}{4 K_{0}} \cdot \frac{K_{0}-\lambda}{1+\lambda-K_{0}}\right\}
$$

whereas the corresponding exact result is

$$
\langle P\rangle=P_{0}\left(1+\frac{1}{2} \frac{G_{k}}{K_{0}}\right)^{-1}
$$

The reason for this failure lies in the very assumptions (7). The second one, regarding the source term is relatively weaker. In fact, in the absence of delayed neutrons, the logarithmic linearization yields the exact result if the source term is better approximated say by iteration (Quabili, 1979). However even with source iteration one cannot reproduce the exact results when delayed neutrons are present (Quabili, 1979). Nevertheless, logarithmic linearization offers wider applicability because it does not require the correlation time to be short or zero and provides one with more accurate results than conventional linearization. A summary of the comparison among the available methods is presented in Table 1 .
We conclude that the two methods described herein fill a gap between the unrestricted but poor conventional linearization and the exact but highly restricted Fokker-Planck theory. At the one end, the logarithmic linearization improves the conventional linearization results at the cost of an added restriction [Gaussian $k(t)]$. At the other end, the stringent requirement that the reactivity noise be Gaussian white has been eased to some extent by Bourret's approximation. In terms of correlation time only, depending on whether it is zero, short or long, one may choose the FokkerPlanck theory, the Bourret's approximation or logarithmic linearization, respectively.

Acknowledgements-The authors are deeply indebted to Professor A. Z. Akcasu for suggesting the problem and extending his help in all stages in this investigation. They are also grateful to the Michigan Memorial Phoenix Project of the University of Michigan for the financial help they received for this investigation.

\section{REFERENCES}

Akcasu A. Z. and Karasulu M. (1976) Ann. nucl. Energy 3,11 .

Akcasu A. Z., Lelleoche G. and Shotkin L. M. (1971) Mathematical Methods in Nuclear Reactor Dynamics, Academic Press, New York.

Ariaratnam S. T. and Graefe P. W. U. (1964) Int. J. Control 1, 239.

Ariaratnam S. T. and Graefe P. W. U. (1965) Int. J. Control 2, 161.

Bourret R. C. (1962) Can. J. Phys. 40, 782.

Dutré W. L. (1967) RCN Report, Proc. of Symp. Statistical Methods in Experimental Reactor Kinetics, Patten, Nederland.

Dutré W. L. and Debosscher A. F. (1977) Nucl. Sci. Engng 62, 355.

Gotoh Y. (1975) Ann. nucl. Energy 2, 119.

Howe M. S. (1971) J. fluid. Mech. 45, 769, 785.

Karmeshu (1978) Ann. nucl. Energy 5, 21.

Karmeshu and Bansal N. K. (1975) Nucl. Sci. Engng 58, 321.

Quabili E. R. (1979) Ph.D. Thesis, University of Michigan.

Redfield A. G. (1965) Advances in Magnetic Resonance (Ed. Waugh J. S.), Vol. 1, Academic Press, New York.

Saito K. (1974) Ann. nucl. Energy 1, 31, 107, 209.

Van Kampen N. G. (1976) Phys. Lett. C24, 171.

Williams M. M. R. (1969) J. nucl. Energy 23, 633.

Williams M. M. R. (1971) Nucl. Sci. Engng 40, 144.

Williams M. M. R. (1974) Random Processes in Nuclear Reactors, Pergamon Press, Oxford. 